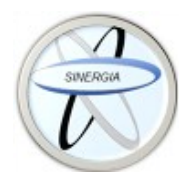

Revista Médica Sinergia

https://doi.org/10.31434/rms.v7i1.750

Vol. 7, Núm. 1, enero 2022, e750

revistamedicasinergia@gmail.com

\title{
Retinopatía diabética
}

\section{Diabetic retinopathy}

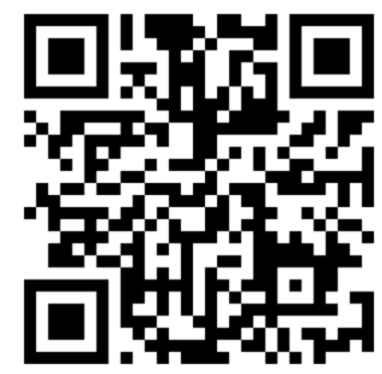

Recibido 02/11/2021
1Dr. Aarón Josué Vargas Ávila
Hospital Calderón Guardia, San José, Costa Rica
(iD) https://orcid.org/0000-0002-4849-1182
${ }^{2}$ Dr. José Ricardo Sojo Padilla
Hospital Calderón Guardia, San José, Costa Rica
(iD) https://orcid.org/0000-0003-0627-8620

${ }^{3}$ Dra. Dayana Campos Bonilla Hospital Calderón Guardia, San José, Costa Rica

(D) https://orcid.org/0000-0001-6414-9371

Corregido $15 / 11 / 2021$
Aceptado 20/11/2021

\section{RESUMEN}

La retinopatía diabética es la complicación más común de la diabetes. En la mayoría de los pacientes, la retinopatía se desarrolla de 10 a 15 años después del diagnóstico de la diabetes. Con el incremento de la prevalecía de la diabetes más personas están en riesgo de sufrir retinopatía. Se espera que para el 2045629 millón de personas padezcan diabetes globalmente. Para el 2010, la retinopatía diabética fue responsable de 3.7 millones de casos problemas visuales y más de 833000 casos de ceguera. El diagnóstico de la retinopatía diabética recae en la detección de lesiones microvasculares. El tratamiento de la retinopatía diabética aun es un desafío, ya que los tratamientos existentes pueden fallar y no alcanzar una mejoría visual clínicamente significativa. Por lo que, hay una necesidad urgente de desarrollar nuevos tratamientos que sean más efectivos.

PALABRAS CLAVE: retinopatía diabética; diabetes; retinopatía diabética proliferativa; retinopatía diabética no proliferativa; edema macular; retina; fotocoagulación.

\section{ABSTRACT}

Diabetic retinopathy is the most common complication of diabetes. In most patients, retinopathy develops 10 to 15 years after the diagnosis of diabetes. With the increasing prevalence of diabetes, more people are at risk for retinopathy. By 2045, 629 million people are expected to have diabetes globally. By 2010 , diabetic retinopathy was responsible for 3.7 million cases of 
visual problems and more than 833,000 cases of blindness. The diagnosis of diabetic retinopathy relies on the detection of microvascular lesions. Treatment of diabetic retinopathy remains a challenge, as existing treatments may fail and fail to achieve clinically meaningful visual improvement. Therefore, there is an urgent need to develop new treatments that are more effective.

KEYWORDS: diabetic retinopathy; diabetes; proliferative diabetic retinopathy; non-proliferative diabetic retinopathy; macular edema; retina; photocoagulation.

\footnotetext{
${ }^{1}$ Médico general, graduado de la Universidad de Iberoamérica de Costa Rica (UNIBE). Cód. MED16018. Correo: ajvargas226@gmail.com

${ }^{2}$ Médico general, graduado de la Universidad de Ciencias Médicas (UCIMED). Cód. MED10090. Correo: jose.sojo@outlook.com

${ }^{3}$ Médica general, graduada de la Universidad de Iberoamérica de Costa Rica (UNIBE). Cód. MED15749. Correo: day22_@hotmail.com
}

\section{INTRODUCCIÓN}

La diabetes mellitus es un término colectivo para desórdenes metabólicos heterogéneos con un metabolismo anormal de los carbohidratos donde su hallazgo principal es la hiperglicemia crónica. La causa es tanto un desorden en la secreción de insulina o un efecto de la insulina o usualmente ambos $(1,2)$.

\section{Diabetes tipo 1}

Entre 2001 al 2009, hubo un incremento del $21 \%$ en el número de jóvenes con diabetes elites tipo 1 en los Estados Unidos (3).

La diabetes tipo 1 se caracteriza por la destrucción autoinmune de las células beta del páncreas, dando un déficit absoluto de la insulina. La diabetes tipo representa del 5 a $10 \%$ de diabetes en adultos (2).

\section{Diabetes tipo 2}

En los Estados Unidos, un estimado de 95\% de cerca de 35 millones de personas viven con diabetes tipo 2. $\mathrm{Y}$ adicionalmente 86 millones de personas padecen prediabetes, poniéndolos en un alto riesgo de desarrollar diabetes tipo 2 (3). La diabetes tipo 2 es por lejos el tipo más común de diabetes en el adulto y es caracterizada por la hiperglicemia usualmente debido a la progresión de la perdida de secreción de la insulina (2).

Independientemente de la zona geográfica, el riesgo de desarrollar diabetes tipo 2 está asociado con bajo nivel socioeconómico, el bajo nivel de educación aumento el riesgo en $41 \%$ al igual que el desempleo $31 \%$ (3).

La retinopatía diabética es una de las causas más grandes de perdida de la visión en el mundo $y$ es la principal causa de impedimento visual en pacientes entre los 25 a 74 años y es la segunda causa de ceguera en Latinoamérica, después de las cataratas $(4,5)$.El desarrollo y la progresión de la retinopatía diabética son causados primariamente por el daño del tejido debido al efecto crónico de la hiperglicemia. El estado de hiperglicemia crónica resulta en cambios estructurales, funcionales $y$ bioquímicos que alteran el metabolismo celular, el flujo de sangre a la retina y la competencia de los capilares de la retina (4).

\section{METODOLOGÍA}

En la selección de artículos para este trabajo de revisión bibliográfica se utilizaron los siguientes criterios de inclusión: artículos en 
los idiomas inglés y español, con fechas de publicación entre los años 2016 a 2021. Se utilizaron bases de datos como Pubmed, Uptodate, The New England Journal of Medicine, Scielo y Cochrane para la búsqueda de información. Además, se utilizaron dos libros de texto: The ophthalmology examinations review y el basic and clinical science course (Retina and vitreous). Para la búsqueda de artículos relacionados se utilizaron los criterios. de búsqueda "retinopatía diabética ", "edema macular", "diabetes".

\section{DEFINICIÓN}

Los criterios diagnósticos para diabetes mellitus son:

- Una glicemia al azar mayor a $200 \mathrm{mg} / \mathrm{dl}$

- Una glicemia en ayunas mayor a 126 $\mathrm{mg} / \mathrm{dl}$

- Una glicemia $2 \mathrm{~h}$ post a dieta mayor a $200 \mathrm{mg} / \mathrm{dl}$

- HbA1c mayor a $6,5 \%$

La retinopatía diabética es la complicación microvascular más común en los pacientes diabéticos, caracterizada por la degeneración de las neuronas de la retina y la neo angiogénesis, además es una de las principales causas de ceguera en el mundo (6-8). La retinopatía diabética clásicamente se ha considerado una enfermedad microvascular de la retina y está caracterizada, en sus últimos estadios, por un crecimiento anormal de los vasos de la retina, lo cual causa hemorragias y tracción de la retina, llevando así a perdida de la visión (8).

\section{CLASIFICACION}

Retinopatía diabética no proliferativa, donde los cambios intravasculares están presentes y no hay desarrollo de tejido fibrovascular extraretinal (9).

La retinopatía diabética proliferativa es definida por la presencia de neovascularización retiniana. Él edema macular diabético es resultado de la permeabilidad vascular anormal y se puede desarrollar en pacientes con cualquier nivel de la retinopatía diabética (9).

El edema macular se define como la presencia de fluido intraretineal y el espesor que envuelve la macula, la parte de la retina responsable de la visión central (10).

La maculopatía diabética es el resultado de alteraciones complejas y multifactoriales de los capilares de la retina en asociación con la diabetes mellitus y se divide en dos formas la maculopatía isquémica y edema macular diabético $(6,7)$.

\section{EPIDEMIOLOGÍA}

La diabetes mellitus es una epidemia global creciente y se espera que afecte 642 millones para el año 2040 (9).

Aproximadamente 400 millones de personas en el mundo tiene diabetes tipo 2 y más de $45 \%$ de ellos tiene retinopatía diabética $(6,7)$.

Actualmente, en los Estados Unidos, se estima que la retinopatía diabética produce entre 12000 a 24000 casos de ceguera por año en adultos de edades entre los 30 a 60 años, siendo así la mayor causa de ceguera en la población trabajadora (11).

Un tercio de la población global con diabetes se estima que tengan retinopatía y de ese grupo se espera que un tercio tengan tratamiento para la retinopatía diabética (9).

- Cerca del $99 \%$ de los pacientes con diabetes tipo 1 y $60 \%$ con diabetes tipo 2 se ha demostrado que pueden tener algún grado de retinopatía diabética (9). 
La retinopatía diabética proliferativa fue encontrada en $50 \%$ en pacientes tipo 1 que tienen 20 años en la enfermedad y $25 \%$ del tipo 2 que tiene 25 años con la enfermedad (9).

La prevalecía de la retinopatía es similar en pacientes con una duración de la diabetes menor a 4 años sin importar el nivel de $\mathrm{HbA1c}(11)$.

Sin embargo, entre pacientes con una duración de la diabetes entre 4 a 8 años y más de 8 años, tienen un aumento marcado en la prevalencia de la retinopatía que en aquellos que tienen una $\mathrm{HbA} 1 \mathrm{c}$ mayor de $7.5 \%$.

\section{ETIOLOGÍA}

Hiperglucemias en un periodo extendido resulta en cambios bioquímicos $\mathrm{y}$ moleculares, entre estos cambios se incluye un aumento en el estrés oxidativo, productos finales de la glicación.

Numerosas anormalidades hematológicas también están asociadas con la instauración y la progresión de la retinopatía, incluido la adhesión plaquetaria, un aumento de la agregación de los eritrocitos y una fibrinólisis defectuosa (9). Cambios en los capilares de la retina como adelgazamiento de la membrana y pérdida selectiva de los pericitos llevan a la oclusión capilar y a la no perfusión retiniana (9).

Estos cambios anatómicos podrían llevar a la oclusión de los capilares de la retina y arteriolas dando como resultado la isquemia retiniana y disrupción de la barrera retiniana, un aumento en la permeabilidad vascular y subsecuentemente edema de la retina (4). Las anormalidades vasculares ocurren tanto en los plexos retinianos superficiales y profundos. Estos cambios empeoran con la severidad de la retinopatía (9).
La hiperglicemia crónica también puede aumentar el flujo sanguíneo en la retina. Este flujo es autorregulado y se mantiene constante hasta que la presión de la arteria central aumenta aproximadamente $40 \%$ sobre su línea de base. Debido a la hiperglicemia este mecanismo de autorregulación se pierde (4).

En los últimos estadios de la enfermedad, la neovascularización de la retina se desarrolla en respuesta al incremento del factor de crecimiento endotelial (9).

Los factores de crecimiento promueven el crecimiento de nuevas venas de venas adyacentes en un intento de revascularizar el tejido enfermo (4).

\section{FACTORES DE RIESGO}

Los factores de riesgo primarios para el desarrollo y la progresión de la retinopatía son:

- Duración de la diabetes

- Control del nivel de glicemia

Factores de riesgo adicionales:

- Hipertensión arterial

- La presencia de otras complicaciones microvasculares

- Dislipidemia

- Embarazo

Todos estos factores de riesgo, en particular la duración de la enfermedad y el nivel de glicemia, son fuertes predictores para el desarrollo y la severidad de la retinopatía (10).

\section{MANIFESTACIONES CLÍNICAS}

\section{Retinopatía diabética no proliferativa}

- Es el estadio inicial de la retinopatía. 
- Incluye micro aneurismas, que son los cambios iniciales visibles, caracterizado por dilatación de los vasos (6).

- Hemorragias intraretinales, que ocurren por la ruptura de capilares débiles (6).

- Exudados duros, que consisten en lipoproteínas que se fugan atreves de vasos retinianos anormales (6).

- Tortuosidades venosas y anormalidades microvasculares intrarerineales (6).

\section{Retinopatía diabética proliferativa}

Podría presentar áreas de hemorragia de la retina y el vítreo, visión oscura, desprendimiento de la retina y todo esto podría provocar ceguera permanente (6).

Edema macular es común en pacientes con retinopatía diabética no proliferativa y se puede desarrollar en cualquier estadio de la enfermedad. Se asocia con el incremento de la permeabilidad de los capilares de la retina y la acumulación de fluidos intraretina (6).

\section{DIAGNÓSTICO}

El diagnóstico inicial puede ser dado mediante la realización de un fondo de ojo o mediante una fotografía de la retina. Este diagnóstico debe ser realizado por aquellos que tengan una adecuada experticia en el método escogido (12). Cuando los exámenes previos han sido normales, las pruebas subsecuentes pueden ser realizados mediante fotografía de la retina. Un fondo de ojo realizado por una persona bien entrenada ha sido el método estándar para realizar el diagnóstico de la retinopatía diabética (12).

En adultos con diabetes tipo 2, se recomienda iniciar la examinación tan pronto al realizar el diagnóstico de diabetes mellitus (12).

En pacientes con diabetes tipo 1, se recomienda iniciar la examinación 5 años después de hacer el diagnóstico de la enfermedad (12).

La detección precoz de la retinopatía diabética resulta fundamental para evitar la ceguera. "La academia Americana de Oftalmología afirma que, si siguen adecuadamente las pautas de tamizaje y se trata oportunamente a los pacientes, la reducción del riesgo de la ceguera se sitúa entre 12 y $28 \% "$ (5).

\section{TRATAMIENTO}

El objetivo del tratamiento de la retinopatía diabética incluye la mejoría en la visión, preservación de la visión y reducción en el ritmo de la progresión y frecuencia de la retinopatía, hemorragia vítrea y edema macular (10).

\section{Control glicémico}

Continúa siendo el cimiento en el cuidado de pacientes con diabetes, y quizás, el factor de riesgo más significativo asociado con el desarrollo y la progresión de la retinopatía diabética (11).

\section{Terapia angiogénica}

- Drogas anti factor de crecimiento endotelial vascular (VEGF)

Los anti VEGF han revolucionado los tratamientos de la retinopatía diabética. Actualmente el tratamiento de la retinopatía diabética consiste en la entrega de anti-VEGF en la retina cuya acción induce a una restricción o inhibición del crecimiento anormal de los 
vasos $(8,13)$. Debido a lo corto de la vida media de los anti VEGF, inyecciones mensuales $O$ bimensuales son necesarios para asegurar la eficacia (14).

Efectos adversos de los anti VEGF: se puede dar más perdida de la visión periférica, más tasa de vitrectomias y mayor desarrollo de edema macular diabético (11).

\section{- Esteroides intraviteos}

Los corticoides intraviteos se han convertido en un tratamiento muy importante del edema macular, especialmente en el edema macular refractario y en pacientes sin respuesta a los anti VEGF (14).

Estos agentes reducen la inflamación mediante dos mecanismos:

Un camino intracelular (genómica) que actúa atreves de la interacción directa con la actividad del ácido desoxirribonucleico (ADN)

Un camino extracelular (no-genómica) que influencia la actividad de los vasos y las células de Müller (15).

Se ha visto que con la triamcinolona intravitea se ha visto una buena eficacia mejorando la agudeza visual $y$ reduciendo el grosor de la retina en pacientes con edema macular en 24 semanas. La eficiencia de la traimcinolona comienza a declinar después de las 24 semanas con mayor riesgo de efectos adversos incluidos presión intraocular elevada y cataratas (14).

\section{- Drogas antiinflamatorias no esteroideos}

Las drogas no esteroideas son las más prescritas, debido a su uso como analgésico, antipirético y antinflamatorios.

Hay enzimas inhibidoras selectivos de ciclooxigenasa - 2 , y reduce la síntesis de prostaglandinas proinflamatoria (15).

Una de las citoquinas proinflamatorias más importantes en el vítreo de los pacientes con retinopatía diabética, es la IL6 y se ha investigado la terapia antiinflamatoria en la retinopatía diabética (14).

\section{- Tratamiento láser}

La fotocoagulación láser ha sido el Gold standard para el tratamiento tanto del edema macular y la retinopatía diabética proliferativa antes que apareciera la terapia con anti VEGF.

Inicialmente, laser de xenón desarrollado por Meyer-Schwickerath fue usado directamente en la ablación de nuevos vasos en la retina (11).

La terapia láser focal en la mácula muestra una efectividad aliviando el edema de la mácula y reduciendo el riesgo de la perdida visual en un $50 \%$ (14).

La terapia láser también puede causar daño en la retina, dando como efectos secundarios pérdida visual central y de la visión nocturna (14).

Complicaciones al usar terapia laser: La complicación más frecuente es la disminución del campo visual debido a la destrucción de foto receptores periféricos.

\section{- Cirugía vitreoretineal}

En la retinopatía diabética, la oclusión de capilares de la retina resulta del crecimiento de neovascularización preretineal de la superficie del disco y de 
los bordes posteriores en las áreas no perfundidas (11).

\section{- Otros agentes terapéuticos}

- Péptido selectivo cardiolipina: la cardiolipina es un fosfolípido dentro de la membrana mitocondrial que podría envolver la apoptosis celular (14).

- Luteína: miembro de la familia de los carotenoides, es un potente antioxidante acumulados en la retina humana (14).

\section{PREVENCIÓN}

Optimizar el tratamiento de condiciones sistémicas en pacientes con diabetes es esencial para prevenir la perdida de la visión. En pacientes con diabetes, esto incluye mantener tanto la glicemia con la presión arterial controlados (10).

Buen control glicémico: mantener un buen control glicemico es la primera medida preventiva en el manejo de la retinopatía diabética. Múltiples estudios han demostrado que disminuyendo el nivel de $\mathrm{HbA} 1 \mathrm{c}$ se reduce el ritmo y la progresión de la retinopatía $(10,11)$. Buen control de la presión arterial: adecuados niveles de presión arterial disminuyen la incidencia de la retinopatía diabética, en algunos estudios, se vio que también hay una disminución en el ritmo de la progresión de la retinopatía diabética y reduce el riesgo de la hemorragia en el vítreo $(10,11)$. Hay cambios de estilo de vida que podrían disminuir la incidencia y el ritmo de progresión de la retinopatía. Ejercicio regular y el aumentar la actividad física podrían llevar a una reducción en la retinopatía diabética (10).

\section{CONCLUSIONES}

El cribaje por retinopatía diabética juega un rol importante en la detección temprana e intervención para prevenir la progresión de la retinopatía diabética.

Abordando los factores de riesgos modificables para retinopatía diabética pueden reducir el riesgo de progresión y complicaciones de la enfermedad.

La obesidad, el pobre control glicémico y el estrés oxidativo han mostrado contribuir al desarrollo de las complicaciones de la retinopatía diabética.

Cribaje regular de los ojos con fundoscopia debería ser parte de la rutina en el manejo de los pacientes con diabetes mellitus.

En las últimas décadas, los anti VEGF se han convertido en la primera línea de tratamiento para la retinopatía diabética proliferativa y el edema macular diabético. Sin embargo, en la práctica clínica, el uso de estos agentes es limitado debido al requerimiento de inyecciones frecuentes, costo y la poca docilidad de los pacientes.

La fotocoagulación juega un rol importante en el tratamiento de la retinopatía diabética como un tratamiento adyuvante.

Los esteroides intraviteos han demostrado beneficios clínicos en el tratamiento del edema macular diabético refractario o en caso de falta de respuesta con la terapia anti VEGF.

Los pacientes diabéticos deben ser educados en las complicaciones oculares que podrían presentar debido a su enfermedad.

\section{Los autores declaran no tener conflicto de interés.}

\section{REFERENCIAS}

1. Petersmann A MWDMULRNMFGea. Definition, classification and diagnosis of diabetes mellitus. Exp Clin Endocrinol Diabetes. 2019;: p. s1-7. 
2. Silvio E Inzucchi MBLM. Clinical presentation, diagnosis, and initial evaluation of diabetes mellitus in adults. UpToDate. 2021.

3. Skyler JS BGBEDTERGLea. Differentiation of diabetes by pathophysiology, natural history and prognosis. Diabetes. 2017; 66(241-55).

4. MD PsS. Diabetic retinopathy: pathogenesis. Uptodate. 2021;(30).

5. Rio Sd. Tamizaje en el diagnostico y prevalencia de retinopatia diabetica en atencion primaria. revista medica chile. 2017; 145(564-571).

6. Rodríguez ML PSMMSDMOÁ. Oxidative stress and microvascular alterations in diabetic retinopathy: future therapies. Oxid Med Cell Longev. 2019.

7. Calderon GD JOHGPSDICZ. Oxidative stress and diabetic retinopathy: development abd treatment. [Online]; 2017. Acceso 02 de octubrede 2021. Disponible en: www.nature.com/eye.

8. Rossino MG CG. Nutraceuticals for the Treatment of Diabetic Retinopathy. nutrients. 2019; 11(771).

9. Colin A. McCannel MAMBGEHPSJKMBCLMRBRMRFSMJKSM.

Retina and Vitreous. En American Academy of Ophthalmology. Basic and clinical sciencie course, Retina and Vitreous. San Francisco ; 2019-2020. p. 91-120.

10. Claire E Fraser MPDJDMARSM. UpToDate. [Online].; 2019. Acceso 02 de octubre de 2021. Disponible en: https://www-uptodate.com.

11. El Rami H BRSJSP. Evidence-Based Treatment of Diabetic Retinopathy. Seminars in Ophthalmology. 2016.

12. David K McCulloch M. UpToDate. [Online]; 2021. Acceso 17 de octubrede 2021. Disponible en: https://www.uptodate.com.

13. Jennifer K. Sun LMJ. The Diabetic Retinopathy Clinical Research Network and its Contributions to the Treatment of Diabetic Retinopathy. Ophthalmic Research. 2019; 62(225-230).

14. Wang W LA. Diabetic Retinopathy: Pathophysiology and Treatments. International Journal of Molecular Sciences. 2018; 19(1816).

15. F. Serrano FMACARSRCC. Diabetic retinopathy, a vascular and inflammatory disease: Therapeutic implications. ELSEVIER. 2019; 11(1086). 